\title{
Thermal modeling of hydrogen storage by absorption in a magnesium hydrides tank
}

\author{
Karim Lahmer $^{1}{ }^{*}$, Rachid Bessaïh ${ }^{1}$, Angel Scipioni ${ }^{2}$, and Mohammed El Ganaoui ${ }^{2}$ \\ 1 Département de Génie Mécanique, (LEAP), Université Constantine 1, Route d'Ain El Bey, Constantine 25000, Algeria \\ 2 Université de Lorraine, Institut Universitaire de Technologie Henri Poincaré de Longwy (LERMAB \& GREEN), \\ 186 Route de Lorraine, 54400 Cosnes-et-Romain, France
}

Received 10 December 2013 / Accepted 13 January 2014 / Published online 14 March 2014

\begin{abstract}
This paper summarizes numerical results of hydrogen absorption simulated in an axisymmetric tank geometry containing magnesium hydride heated to $300{ }^{\circ} \mathrm{C}$ and at moderate storage pressure $1 \mathrm{MPa}$. The governing equations are solved with a fully implicit finite volume numerical scheme used by a commercial software FLUENT. The effect of the different kinetic reaction equations modeling hydrogen absorption was studied by the introduction of a specific subroutine at each time step in order to consider which one will provide results close to available experimental results. Spatial and temporal profiles of temperature and concentration in hydride bed are plotted. Results show that suitable method for our two-dimensional study is a CV-2D technique because it generates the smallest error especially during the beginning of the reaction. Also, its computational time is the shortest one compared to the other methods.
\end{abstract}

Key words: Hydrogen storage, Magnesium hydrides, CFD simulation, Absorption kinetic equations.

\section{Nomenclature}

$C_{p} \quad$ specific heat, $\mathrm{J} / \mathrm{kg} \mathrm{K}^{-1}$

$C_{\mathrm{a}} \quad$ kinetic coefficient, $\mathrm{s}^{-1}$

$\Delta H \quad$ molar enthalpy of reaction at standard conditions, $\mathrm{J} / \mathrm{mol}$

$K \quad$ permeability, $\mathrm{m}^{2}$

$P \quad$ hydrogen pressure, $\mathrm{Pa}$

$P_{\text {eq }}(T) \quad$ equilibrium pressure, $\mathrm{Pa}$

$R \quad$ universal gas constant $=8.314 \mathrm{~J} / \mathrm{mol} \mathrm{K}$

$S \quad$ source term of reaction, $\mathrm{mol} / \mathrm{m} \mathrm{s}$

$\Delta S \quad$ molar entropy of reaction at standard conditions, $\mathrm{J} / \mathrm{mol} \mathrm{K}$

$w t \quad$ maximum weight percentage of hydrogen in the material, $\%$

\section{Greek symbols}

$\begin{array}{ll}\alpha & \text { absorption rate } \\ \lambda & \text { thermal conductivity, } \mathrm{W} / \mathrm{m} \mathrm{K} \\ \varepsilon & \text { porosity } \\ \rho & \text { density, } \mathrm{kg} / \mathrm{m}^{3} \\ \mathrm{~d} \alpha / \mathrm{d} t & \text { hydriding velocity, } \mathrm{s}^{-1}\end{array}$

*e-mail: lahmer.karim@sonelgaz.dz

\section{Subscripts and superscripts}

$\begin{array}{ll}\text { e } & \text { energy } \\ \text { eff } & \text { effective } \\ \text { eq } & \text { balance } \\ \mathrm{g} & \text { gas } \\ \mathrm{H} & \text { hydrogen } \\ \mathrm{M} & \text { metal } \\ \mathrm{m} & \text { mass } \\ \mathrm{MH} & \text { metal hydride } \\ \mathrm{i} & \text { initial }\end{array}$

\section{Introduction}

Because of the huge amounts of dangerous gases emitted by industry, the predicted shortage of fossil fuels and the announced general climate change, SOFC fuel cells running with hydrogen could be the solution to the recurring problem of intermittent renewable energy. These kinds of systems are particularly interesting for electricity production in isolated areas, for the daily stabilization of electricity demand or as security high power kits. However, the major obstacle to the rapid growth of this technology is the hydrogen storage in the most compact volume. One of the most promising methods is that of storing hydrogen in metal hydrides. Indeed, magnesium hydride is a very good substance for reversible hydrogen 
storage because of its highest capacity of storage $\left(7.6 \% \mathrm{~m} \mathrm{H}_{2}\right)$ compared to the others such as $\mathrm{TiVCrH}_{6}\left(2 \% \mathrm{~m} \mathrm{H}_{2}\right), \mathrm{FeTiH}_{1.95}$ $\left(1.6 \% \mathrm{~m} \mathrm{H}_{2}\right), \quad \mathrm{LaNi}_{5} \mathrm{H}_{6.7}\left(1.5 \% \mathrm{~m} \mathrm{H}_{2}\right)$. Its volume capacity $\left(111 \mathrm{~kg} / \mathrm{m}^{3}\right)$ is greater than that of liquid hydrogen $\left(71 \mathrm{~kg} / \mathrm{m}^{3}\right)$, that's why it would be used in a large scale in the few next years. The hydrogen storage in a solid form offers significant advantages, like reducing the tank dimensions, resulting chemical reactions running at moderate temperatures $300{ }^{\circ} \mathrm{C}$ and at pressures about 1-10 bars; also, hydrogen density in the solid form is more important than the one in molecular form. Finally, this type of storage allows better safety compared to conventional methods such as compression or liquefaction of hydrogen.

Many experimental and computational studies were realized in order to improve the hydrogen tank performances. Jemni and Nasrallah [1] realized one of the first numerical studies of 2Dhydrogen reactor. The results showed the importance of the geometry, inlet pressure and inlet temperature choice. Kaplan et al. [2] presented a mathematical model for hydrogen storage in a metal hydride bed; the team concluded that a rapid charge needs efficient cooling. Muthukumar et al. [3-9] made a parametric investigation of a metal hydride hydrogen storage device, they showed that overall increasing heat transfer coefficient is not beneficial. Phate et al. [4] carried out a computational analysis of a cylindrical metal hydride bed; their conclusion is that the concentration gradient in the bed is the major driving force of hydrogen flow in the bed. Marty et al. [5-14] added an experimental validation to the computational simulations of the hydrogen storage tank with metal hydrides; their goal was to obtain performances according to the objectives imposed by a stationary cogeneration system. Askri et al. [6] made a numerical investigation of heat and mass transfer of a 3D annular tank. Results showed that the use of fins enhances heat transfer and consequently $40 \%$ improvement of the time required for $90 \%$ storage can be achieved over the case without fins. A CFD model for simulating hydrogen storage in an activated carbon tank was described by Chahine et al. [7]; this model showed that the amount of adsorbed hydrogen is greater than that of the compressed gas hydrogen.

Because of these many coupled phenomena, numerical simulation allows us to anticipate and understand the evolution of the hydrogen storage reaction. In addition, the numerical tool will save considerable time for the optimization of hydrogen tank design. The industrial software FLUENT was used to perform numerical simulations. One of the advantages of this code consists of the important part devoted to the modeling of thermodynamics and kinetics reaction between a gas and a porous medium. A file called (UDF: User Defined Function) in $\mathrm{C}$ grouping models was realized and included in the calculations. The purpose of this work is to find the best reaction kinetics equation which is the most adapted for phenomena modeling of the hydrogen absorption.

\section{Geometry and mathematical formulation}

The tank geometry (Figure 1) consists of a domain with $(L=450 \mathrm{~mm}) \times(H=7.5 \mathrm{~mm})$ dimensions. It is surrounded by an aluminum shell which equalizes the temperature. Also,

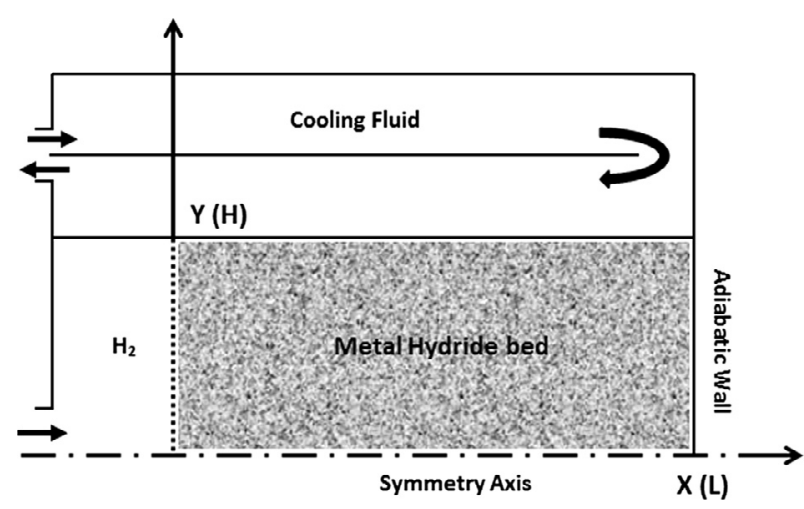

Figure 1. Schematic of a basic tank.

it is cooled by a process which ensures effective further reactions. Hydrogen enters from a lateral left side. The activated magnesium hydride porous powder (Metal alloy) is retained on the other side by a thin filter. The right side wall is considered adiabatic.

In order to simplify the model, some assumptions are adopted. First, hydrogen is considered as an ideal gas between the hydride pores and its generated flow before the absorption phase is negligible. Then, the hydrogen temperature is locally the same as that of the powder, the flow is laminar between the powder grains, the radiative transfer in the porous medium is neglected, the pressure and friction energies are negligible, the inlet temperature is constant. Finally, the resolution method used is the finite volume in a two-dimensional, axisymmetric, laminar regime and at unsteady flow.

The governing equations of the phenomenon are given in the following sections.

\subsection{The equations of mass balance and Darcy}

- Continuity equation in the gas phase

$$
\varepsilon_{\mathrm{M}} \frac{\partial \rho_{\mathrm{g}}}{\partial t}+\operatorname{div}\left(\rho_{\mathrm{g}} \cdot \vec{U}\right)=-S_{\mathrm{m}} \cdot M_{\mathrm{H} 2} .
$$

- Continuity equation in the solid phase

Mass transfer is taken into account by Fluent with the continuity equation and the Darcy one:

$$
\varepsilon_{\mathrm{MH}} \frac{\partial \rho_{\mathrm{MH}}}{\partial t}=S_{\mathrm{m}} \cdot M_{\mathrm{H} 2} .
$$

The term source of hydrogen mass depends on the reaction rate and can be expressed:

$$
S_{\mathrm{m}}=\rho_{\mathrm{MH}} \cdot w t(1-\varepsilon) \frac{\mathrm{d} \alpha}{\mathrm{d} t} .
$$

The Darcy equation is: $\vec{\nabla} P_{\mathrm{H} 2}=-\frac{\mu}{K} \cdot \vec{U}$.

This expression is the balance between viscous friction and pressure gradient where $(K)$ is the permeability of the metal hydride. Considering a high porosity of the material and a low velocity of the flow, we can suppose that: $\vec{\nabla} P_{\mathrm{H} 2}=0$. 


\subsection{The energy equation}

The energy conservation equation simplified and solved by fluent in both phases is:

$$
\begin{aligned}
& \varepsilon \rho_{\mathrm{g}} C_{p_{\mathrm{g}}} \frac{\partial T}{\partial t}+(1-\varepsilon) \rho_{\mathrm{s}} C_{p_{\mathrm{s}}} \frac{\partial T}{\partial t}+\varepsilon \rho_{\mathrm{g}} C_{p_{\mathrm{g}}} \nabla(T \cdot \vec{U}) \\
& =\nabla\left(\lambda_{\mathrm{eff}} \nabla T_{\mathrm{MH}}\right)+S_{\mathrm{e}} .
\end{aligned}
$$

- Since the solid medium $(\mathrm{s})+\mathrm{gas}(\mathrm{g})$ is treated as a continuous medium with:

$$
\begin{aligned}
& \rho_{\mathrm{MH}} C_{p_{\mathrm{MH}}}=\varepsilon \rho_{\mathrm{g}} C_{p_{\mathrm{g}}}+(1-\varepsilon) \rho_{\mathrm{m}} C_{p_{\mathrm{m}}}=\sum_{i} \varepsilon_{\mathrm{i}} \rho_{\mathrm{i}} C_{p_{\mathrm{i}}} \\
& \lambda_{\mathrm{eff}}=\varepsilon \lambda_{\mathrm{g}}+(1-\varepsilon) \lambda_{\mathrm{m}} .
\end{aligned}
$$

So, to the absorption source term of the energy equation taken into account through a module in Fluent UDF is:

$$
S_{\mathrm{e}}=\frac{\Delta H}{M_{\mathrm{H} 2}} S_{\mathrm{m}} \text {. }
$$

The material thermal parameters are a function of the powder compactness, temperature and hydrogen inlet pressure and absorption rate. Also, a good knowledge of the thermal conductivity of the activated powder is particularly necessary to obtain perfect simulation accuracy. This value was already obtained experimentally and taken into account such as: $\lambda_{\text {eff }}=$ $0.48 \mathrm{~W} / \mathrm{m} / \mathrm{K}$.

\subsection{Initial and boundary conditions}

\subsubsection{Initial conditions}

Initially, the powder temperature, the gas pressure and the hydride density of the reaction bed are considered uniform through the tank and the system was assumed under the P-c-T equilibrium

$$
T_{\mathrm{S}}=T_{\mathrm{H} 2}=T_{\mathrm{i}} ; \quad P_{\mathrm{H} 2}=P_{\mathrm{i}} ; \quad \rho_{\mathrm{H} 2}=\rho_{\mathrm{i}} .
$$

\subsubsection{The boundaries conditions}

The boundary conditions taken into consideration are:

- Hydrogen is supplied axially to the hydride bed through a porous filter.

$$
\left.\frac{\partial T_{\mathrm{H} 2}}{\partial X}\right|_{x=0}=0 ;\left.\quad \frac{\partial P_{\mathrm{g}}}{\partial X}\right|_{x=0}=0
$$

- Wall with heat transfer at the top of tank (forced convection by cold fluid)

$$
-\left.\lambda_{\text {eff }} \frac{\partial T_{\mathrm{MH}}}{\partial Y}\right|_{y=H}=h\left(T_{\mathrm{MH}}-T_{\infty}\right) ;\left.\frac{\partial P_{\mathrm{g}}}{\partial Y}\right|_{y=H}=0 .
$$

- Adiabatic right tank wall:

$$
\left.\frac{\partial T_{\mathrm{MH}}}{\partial X}\right|_{x=L}=0 ;\left.\quad \frac{\partial P_{\mathrm{H} 2}}{\partial X}\right|_{x=L}=0
$$

Table 1. Different modeling mechanisms equations [8].

\begin{tabular}{ll}
\hline \multicolumn{1}{c}{ Models equation } & \multicolumn{1}{c}{ Method } \\
\hline$k t=\alpha$ & Chemisorption \\
$k t=[-\ln (1-\alpha)]^{1 / 2}$ & 2D/Johnson-Mehl-Avrami-Kolmogorov: \\
& two-dimensional growth of \\
& existing nuclei with constant interface \\
& velocity (JMAK-2D) \\
$k t=1-\ln (1-\alpha)^{1 / 3}$ & 3D/Contracting Volume with \\
& constant interface velocity (C V-3D) \\
$k t=1-\ln (1-\alpha)^{1 / 2}$ & 2D/Contraction Volume with \\
& constant interface velocity (C V-2D) \\
\hline
\end{tabular}

with: $k(T, P)=C_{\mathrm{a}} \mathrm{e}^{\frac{-E_{\mathrm{a}}}{R T} \frac{P-P_{\mathrm{eq}}}{P_{\mathrm{eq}}}}$ (the Arrhenius law) and $E_{\mathrm{a}}, C_{\mathrm{a}}, \mathrm{R}$ are extracted values from the experiment study [11].

\subsection{Modeling of thermodynamics and kinetic reaction}

The modeling of thermodynamics and kinetics reaction permits the calculation of the spatial and temporal evolution of absorption rate and hydriding velocity into the material. These laws are included in the UDF that allows an iterative calculation at the end of each time step $\Delta t$ ).

There are different mechanisms that can model the reaction kinetics. In this study, we have compared the results of these models in order to know the best mechanism which closely approximates the results obtained from the experiences of the real phenomenon. The considered mechanisms are shown in Table 1.

The terms of the absorption rate $(0<\alpha<1)$ and the hydriding velocity $\frac{\mathrm{d} \alpha}{\mathrm{d} t}$ are derived from the models equations of different reaction.

The law which defines the thermodynamic equilibrium pressure $\left(P_{\text {eq }}\right)$ between the two domains of existence of $\mathrm{Mg}$ and $\mathrm{MgH}_{2}$ is the Van't Hoff law. It is given by the following expression:

$$
P_{\text {eq }}(T)=P^{\circ} \mathrm{e}^{\left[\frac{\Delta H}{R T}-\frac{\Delta S}{R}\right]},
$$

where $P^{\circ}=P_{\text {atm }}, \Delta H$ : the absorption enthalpy, $\Delta S$ : the entropy of the reaction.

\section{Validation}

Our results were compared with the published experimental data. In fact, we performed FLUENT simulations and confronted them with those available in relevant studies such as [12]. Figure 2 shows the profile of the average temperature of the metal hydride bed as a function of elapsed hydrogen absorption time. Figures 3 and 4 present the comparison with the average temperature contours and the hydrogen capacity with the contribution of [13]. Our computational results showed good agreement with those available in literature.

This study aims to model the heat transfer and reaction kinetics during hydrogen loading magnesium hydride tank. FLUENT industrial software was chosen to model this problem. It uses the finite volume method to discretize the equations of 


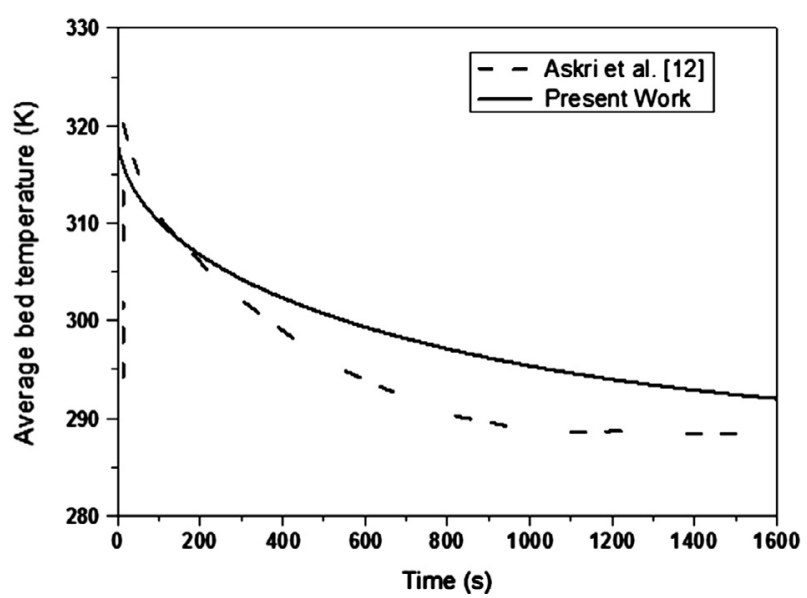

Figure 2. Absorption average temperature profiles with $P_{\mathrm{H} 2}=3 \mathrm{MPa}$ and $\Delta t=0.1 \mathrm{~s}$.

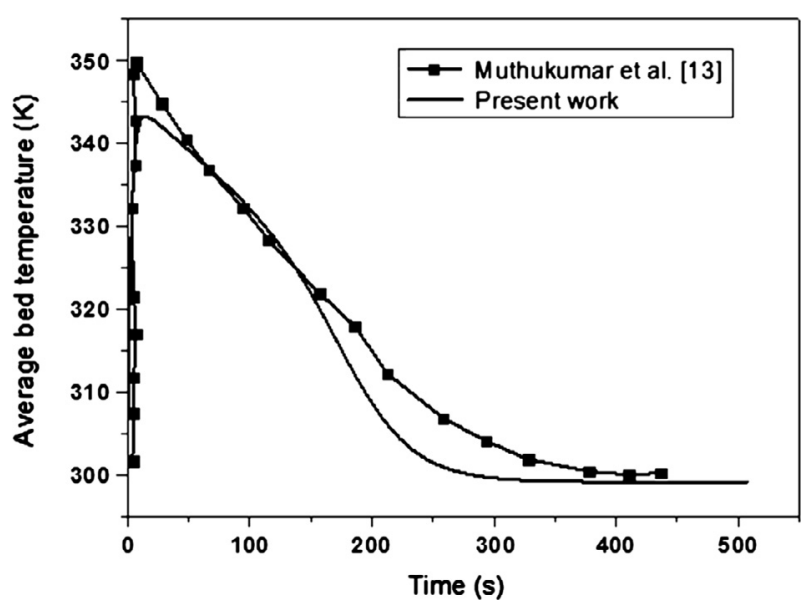

Figure 3. Absorption average temperature profiles with $P_{\mathrm{H} 2}=2 \mathrm{MPa}$ and $\Delta t=0.1 \mathrm{~s}$.

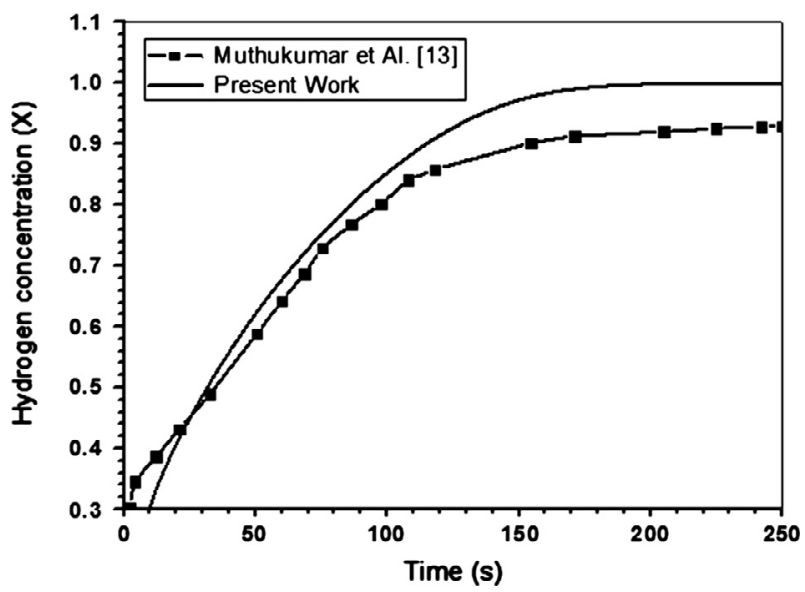

Figure 4. Variation of hydrogen concentration with time.
Table 2. Main parameter values used in computations [10].

\begin{tabular}{|c|c|}
\hline \multicolumn{2}{|c|}{ Input boundary conditions } \\
\hline $\begin{array}{l}T_{\mathrm{i}}=573 \mathrm{~K} \\
T_{\mathrm{f}}=300 \mathrm{~K} \\
P_{\mathrm{i}}=1 \mathrm{MPa} \\
h=10^{3} \mathrm{~W} / \mathrm{m}^{2} \mathrm{~K}\end{array}$ & $\begin{array}{l}\text { Initial bed temperature } \\
\text { Cold fluid temperature } \\
\text { Supply pressure } \\
\text { Heat transfer coefficient }\end{array}$ \\
\hline \multicolumn{2}{|c|}{ Properties of metal alloy } \\
\hline $\begin{array}{l}\rho_{\mathrm{m}}=1945 \mathrm{~kg} / \mathrm{m}^{3} \\
C p_{\mathrm{MH}}=1545 \mathrm{~J} / \mathrm{kg} \mathrm{K} \\
\lambda_{\mathrm{eff}}=0.48 \mathrm{~W} / \mathrm{m} \mathrm{K} \\
\varepsilon=0.77 \\
E_{\mathrm{a}}=130 \mathrm{~kJ} / \mathrm{mol} \mathrm{H} 2 \\
\Delta S=135.6 \mathrm{~J} / \mathrm{mol} \mathrm{H} 2 \mathrm{~K} \\
\Delta H=75 \mathrm{~kJ} / \mathrm{mol} \mathrm{H} 2\end{array}$ & $\begin{array}{l}\text { Metal density } \\
\text { Specific heat of metal } \\
\text { Effective thermal conductivity } \\
\text { Porosity } \\
\text { Activation energy } \\
\text { Entropy of formation } \\
\text { Enthalpy of formation }\end{array}$ \\
\hline \multicolumn{2}{|c|}{ Properties of hydrogen } \\
\hline $\begin{array}{l}\lambda_{\mathrm{g}}=0.127 \mathrm{~W} / \mathrm{m} \mathrm{K} \\
C p_{\mathrm{g}}=14283 \mathrm{~J} / \mathrm{kg} \mathrm{K} \\
\rho_{\mathrm{g}}=0.0838 \mathrm{~kg} / \mathrm{m}^{3}\end{array}$ & $\begin{array}{l}\text { Thermal conductivity of hydrogen } \\
\text { Specific heat of hydrogen } \\
\text { Hydrogen density }\end{array}$ \\
\hline \multicolumn{2}{|c|}{ Constants } \\
\hline $\begin{array}{l}R=8.314 \mathrm{~J} / \mathrm{mol} \mathrm{K} \\
k_{\mathrm{c}}=10^{11} \mathrm{~s}^{-1}\end{array}$ & $\begin{array}{l}\text { Universal gas constant } \\
\text { Reaction constant }\end{array}$ \\
\hline
\end{tabular}

the mathematical model. Then, it solves in an unsteady case the equations of mass, momentum and energy and includes the kinetic reaction differential equations with the appropriate physical parameters. These physical parameters used in the numerical simulations are presented in Table 2.

\section{Results and discussion}

Computations were carried out under windows with an Intel core i5 processor. The simulations were executed on several grids and time steps ranging from $0.01 \mathrm{~s}$ to $1 \mathrm{~s}$. The mesh corresponding to $396 \times 210$ nodes and time step $\Delta t=10^{-2} \mathrm{~s}$ was therefore adopted for all numerical simulations, in order to optimize the calculating time and the convergence criterion. At the end of the computations, we observed (Figure 5) that the hydrogen absorption phenomenon which starts with preheated discharged tank from $573 \mathrm{~K}$ at supply pressure of $10 \mathrm{MPa}$ causes increase in the metal hydride temperature during the first ten seconds of loading and reaches different maximum temperatures from $625 \mathrm{~K}$ to $670 \mathrm{~K}$ depending on the used kinetic reaction model. This quick temperature increase during tank loading is due to absorption exothermic reactions and hydrogen compression process which produce heat. Then, it slows down gradually and becomes equal to the cooling temperature of $300 \mathrm{~K}$.

Figure 6 shows stratified isothermal contours when $x=50 \mathrm{~mm}$ at $52 \mathrm{~s}$ using the kinetic reaction method of JMAK. In addition, significant temperature gradients were recorded between the tank surface and its center. These thermal gradients are axial and radial. The temperature reaches a maximum value at the center of the tank, and is lower in the area close to the wall. Finally, the results showed little differences between temperature contours when changing kinetic reaction models.

Figure 7 shows hydrogen capacity profiles with different kinetic reaction equations. Indeed, the hydrogen capacity 


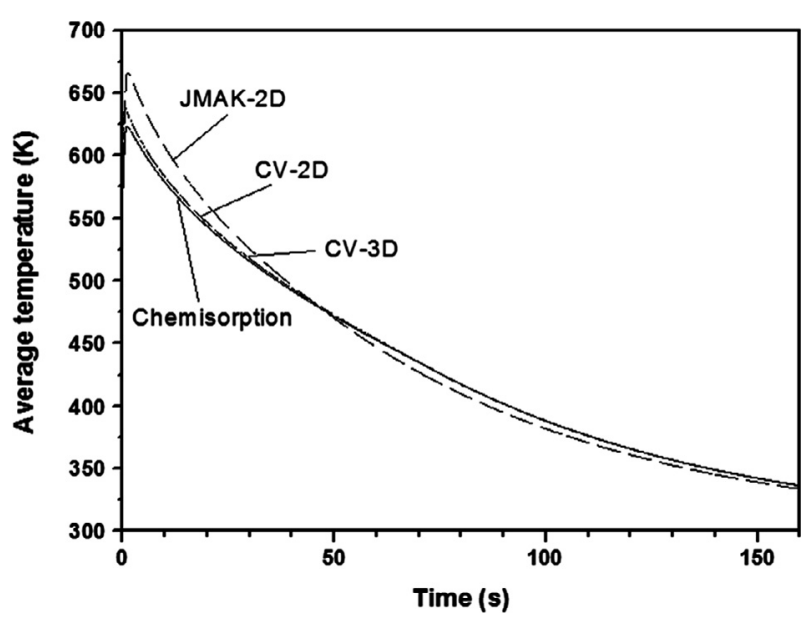

Figure 5. Absorption average temperature profiles with the different kinetic equations methods with $\Delta t=0.01 \mathrm{~s}$ and at supply pressure of $1 \mathrm{MPa}$.

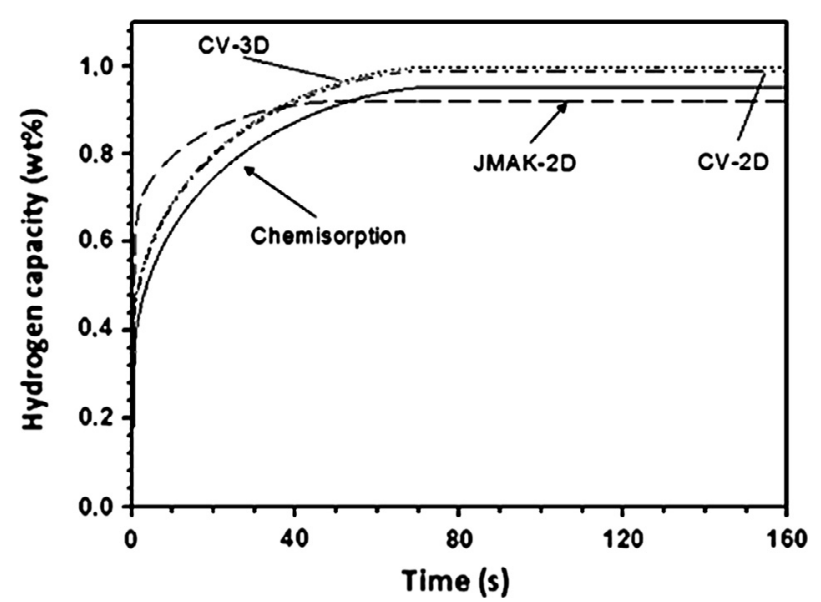

Figure 7. Hydrogen storage capacity profiles at supply pressure of $1 \mathrm{MPa}$ with different methods.

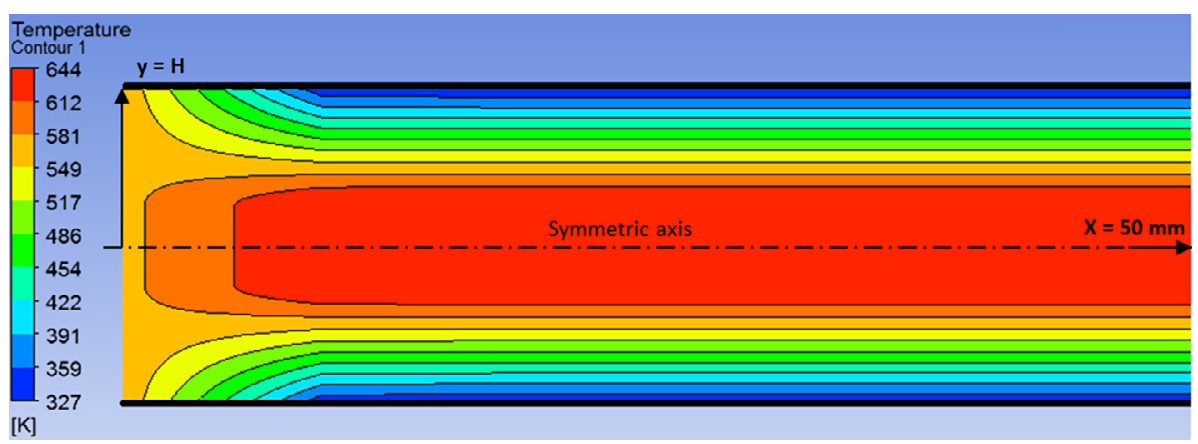

Figure 6. Average bed temperature contours at absorption time $t=52 \mathrm{~s}$ and $x=50 \mathrm{~mm}$ with JMAK method (horizontal line is the symmetric axis of geometry).

increases gradually during the first 73 s. Then, it becomes constant after reaching its maximum value.

Since the JMAK method is the mostly used in comparative studies with experimental data, the obtained results show that the CV-2D is the most suitable method in such a case as compared to the other models.

\section{Conclusion}

In this numerical study, thermal aspects of a two-dimensional metal hydride tank are explored by formulating transient heat transfer by conduction. Four different models for kinetic reaction in porous bed (metal hydride) were introduced by an UDF at Fluent simulations. Fluent uses the finite volume method to discretize the equations generated from the mathematical model. The validation was realized when comparing the obtained results with the experimental data and a good agreement has been observed.

Results have shown an important and quick generated heat at the beginning of the hydrogen absorption process into metal hydride. These temperature gradients between the center and the surface of the tank are axial and radial. Also, little differences between temperature contours were noticed when chang- ing kinetic reaction models. Also, cooling tank becomes necessary to allow the continuation of hydrogen loading.

The use of different equations to model the kinetics of reaction has been found to produce almost similar results. However, the most suitable method for our two-dimensional study is a CV-2D technique because it generates the smallest error especially during the beginning of the reaction. Also, its computational time is the shortest one compared to the other methods (e.g., the JMAK)

Those results are the first step in our efforts to optimize the hydrogen storage tank. The Modeling continues in a 3D perspective and can be extended to predict the best tank cooling mode.

Acknowledgements. Special thanks to "Longwy IUT - France" staff for their welcoming, support and their important contribution to this work.

\section{References}

1. Jemni A, Ben Nasrallah S. 1995. Study of two dimensional Heat and mass transfer during absorption in a metal hydrogen reactor. International Journal of Hydrogen Energy, 20(1), 43-52. 
2. Dogan A, Kaplan Y, Veziroglu TN. 2004. Numerical investigation of heat and mass transfer in a metal hydride bed, Applied Mathematics and Computation, 150, 169-180.

3. Muthukumar P, Madhavakrishna U, Dewan A. 2007. Parametric studies on a metal hydride based hydrogen storage device. International Journal of Hydrogen Energy, 32, 4988-4997.

4. Phate K, Maiya MP, Murthy SS. 2007. Simulation of transient heat and mass transfer during hydrogen sorption in cylindrical metal hydride beds. International Journal of Hydrogen Energy, 32, 1969-1981.

5. Botzung M, Chaudourne S, Gillia O, Perret C, Latroche M, Percheron-Guegan A, Marty P. 2008. Simulation and experimental validation of a hydrogen storage tank with metal hydrides. International Journal of Hydrogen Energy, 33, 98104.

6. Askri F, Ben Salah M, Jemni A, Ben Nasrallah S. 2009. Heat and mass transfer studies on metal-hydrogen reactor filled with $\mathrm{MmNi}_{4.6} \mathrm{Fe}_{0.4}$. International Journal of Hydrogen Energy, 34, 6705-6711.

7. Ye F, Xiao J, Hu B, Bénard P, Chahine R. 2012. Implementation for model of adsoptive hydrogen storage using UDF in fluent. Physics Procedia, 24, 793-800.

8. Varin R, Czujkon T, Wronski ZS. 2009. Nanomaterials for Solid State Hydrogen Storage Fuel cells and hydrogen energy. Springer, ISBN 978-0-387-77711-5.

9. Muthukumar P, Prakash Maiya M, Murthy SS, Vijay R, Sundaresan R. 2008. Tests on mechanically alloyed $\mathrm{Mg}_{2} \mathrm{Ni}$ for hydrogen storage. Journal of Alloys and Compounds, 452, 456461.

10. Chaise Albin. 2008. Etude expérimentale et numérique des réservoirs à hydrures de magnésium. Ph.D. dissertation, Material Physics speciality, Joseph Fourier University.

11. Chaise A, Marty P, de Rango D, Fruchart D. 2009. A simple criterion for estimating the effect of pressure gradients during hydrogen absorption in a hydride reactor. International Jounal of Heat and Mass Transfer, 52, 4564-4572.

12. Askri F, Ben Salah M, Jemni A, Ben Nasrallah S. 2009. Heat and mass transfer studies on metal-hydrogen reactor filled with $\mathrm{MmNi}_{4.6} \mathrm{Fe}_{0.4}$. International Journal of Hydrogen Energy, 34, 6705-6711.

13. Muthukumar P, Venkata Ramana S. 2009. Numerical simulation of coupled heat and mass transfer in metal hydride-based hydrogen storage reactor. Journal of Alloys and Compounds, 472, 466-472.

14. Botzung M, Chaudourne S, Gillia O, Perret C, Latroche M, Percheron-Guegan A, Marty P. 2008. Simulation and experimental validation of a hydrogen storage tank with metal hydrides. International Journal of Hydrogen Energy, 33, 98104.

15. Yang F, Zhang Z. Febuary2011. Simulation studies on the coupling process of heat/mass transfer in a metal hydride reactor. Mass Transfer in Multiphase Systems and its Applications, Pub. 11

16. Gambini M, Manno M, Vellini M. 2008. Numerical analysis and performance assessment of metal hydride-based hydrogen storage systems. International Journal of Hydrogen Energy, $33,6178-6187$.

17. Hermosilla-Lara G, Momen G, Marty PH, Le Neindre B, Hassouni K. 2007. Hydrogen storage by adsorption on activated carbon: investigation of the thermal effects during the charging process. Investigation of the International Journal of Hydrogen Energy, 32, 1542-1553.

18. Marty P, Fourmigue JF, de Rango P, Fruchart D, Charbonnier J. 2006. Numerical simulation of heat and mass transfer during the absorption of hydrogen in a magnesium hydride. Energy Conversion and Management, 47, 3632-3643.

Cite this article as: Lahmer $\mathrm{K}$, Bessaïh $\mathrm{R}$, Scipioni A \& El Ganaoui M: Thermal modeling of hydrogen storage by absorption in a magnesium hydrides tank. Int. J. Simul. Multisci. Des. Optim., 2014, 5, A21. 